\title{
A sufficient condition for bicolorable hypergraphs
}

\author{
David Défossez ${ }^{1}$ \\ ${ }^{1}$ Laboratoire Leibniz-Imag, 46 avenue Félix Viallet, 38031 Grenoble Cédex, France. E-mail: david.defossez@imag.fr
}

In this note we prove Sterboul's conjecture, that provides a sufficient condition for the bicolorability of hypergraphs.

Keywords: hypergraphs, coloring, Sterboul's conjecture

In [2], Fournier and Las Vergnas gave a sufficient condition for the bicolorability of hypergraphs. Their theorem was a weaker form of a conjecture due to Sterboul, that we prove here. These facts are reproduced in [1] and [3].

A hypergraph is a pair $H=(V, \mathcal{E})$, where the elements of $V$ are the vertices, and the elements of $\mathcal{E}$ are subsets of $V$ and are called the edges. A function $c: V \rightarrow\{1,2\}$ is called a bipartition of $V$, and for $x \in V$, we call $c(x)$ the color of $x$. If $c$ is such that for any $e \in \mathcal{E}$ with $|e| \geq 2$ both colors occur, then $c$ is called a bicoloration of $H$. If only one color occurs, the edge is said to be monochromatic. If a hypergraph admits a bicoloration we say that it is bicolorable.

A sequence $\left(x_{1}, e_{1}, x_{2}, \ldots, e_{k}, x_{1}\right)$, where the $e_{i}$ 's are distinct edges, the $x_{i}$ 's are distinct vertices, and $k \geq 3$, is said to be a cycle if $x_{i} \in e_{i-1} \cap e_{i}$ for $i=2, \ldots, k$ and $x_{1} \in e_{1} \cap e_{k}$. A cycle is said to be odd if it has an odd number of edges.

An odd cycle $\left(x_{1}, e_{1}, x_{2}, \ldots, e_{k}, x_{1}\right)$ such that two non-consecutive edges are disjoint and $\left|e_{i} \cap e_{i+1}\right|=1$ for $i=1,2, \ldots, k-1$, is called a Sterboul cycle. If a hypergraph $H$ has no Sterboul cycle, it is said to be a Sterboul hypergraph.

Then we can word Sterboul's conjecture as follows:

Theorem 1 If $H$ is a Sterboul hypergraph, then $H$ is bicolorable.

Proof: The proof works by induction on the number of edges.

When the hypergraph has no edge, the theorem clearly holds.

The general step assumes that we have a hypergraph $H=(V, \mathcal{E})$ and $e_{0} \in \mathcal{E}$ such that $H \backslash e_{0}=$ $\left(V, \mathcal{E} \backslash e_{0}\right)$ has a bicoloration $c: V \rightarrow\{1,2\}$.

We can assume that $e_{0}$ has size at least 2 , and that $c$ leaves $e_{0}$ monochromatic, or else we have nothing to do.

1365-8050 @ 2005 Discrete Mathematics and Theoretical Computer Science (DMTCS), Nancy, France 
Now we use the following algorithm to transform the bipartition $c$ into a bicoloration of $H$. The algorithm switches successively the colors of some vertices of $H$ that are contained in a monochromatic edge in the current bipartition. It constructs an arborescence $G_{0}=\left(V_{0}, E_{0}\right)$ and a mapping $g: V_{0} \rightarrow \mathcal{E}$ that keep track of the running of the algorithm: the vertices of $G_{0}$ are those whose colors were switched, and $g$ associates a vertex with the monochromatic edge that caused its color switch.

The vertices are chosen with a DFS (Depth-First Search) method, and to do so the algorithm uses a LIFO (Last In First Out) stack $\mathcal{P}$ that contains the set of vertices whose colors have been switched, and so that might be in a monochromatic edge. top $(\mathcal{P})$ returns the last vertex entered in $\mathcal{P}$; $\operatorname{drop}(\mathcal{P})$ removes top $(\mathcal{P})$ out of $\mathcal{P}$; and put $(x, \mathcal{P})$ enters a new vertex $x$ in $\mathcal{P}$.

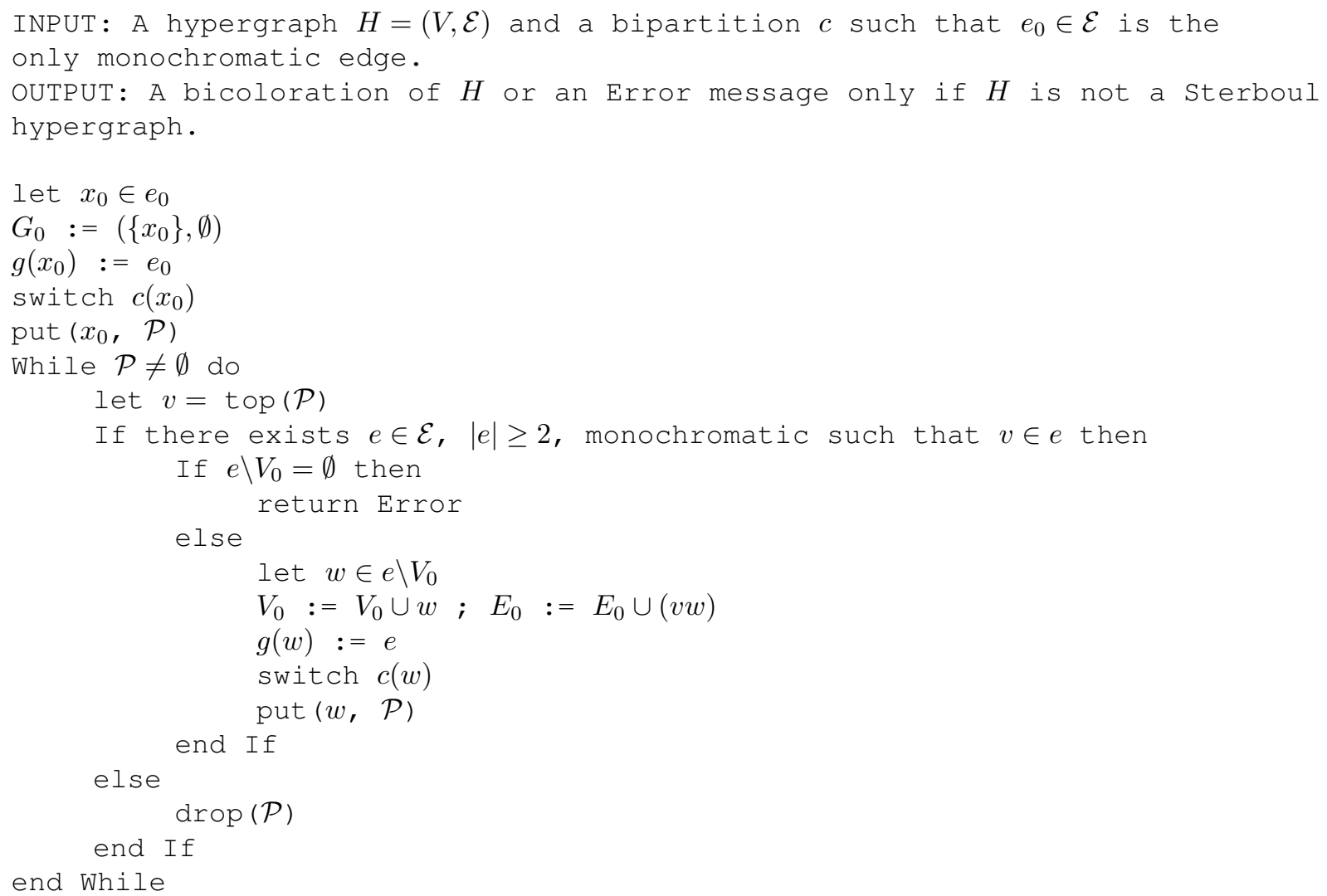

First we remark that $G_{0}$ is indeed an arborescence since the end point of each new arc of $G_{0}$ is a new vertex. Then for a given $x \in V_{0}$ there is a unique path in $G_{0}$ from $x_{0}$ to $x$. Moreover when $x$ is at the top of $\mathcal{P}$, then $\mathcal{P}$ contains exactly the vertices of that path (because $\mathcal{P}$ is a LIFO stack).

We can also remark that if the algorithm does not return Error, then at each iteration either a new vertex is put into $\mathcal{P}$, or a vertex is dropped out of $\mathcal{P}$. Since a vertex appears at most once in $G_{0}$ and thus can be put at most once in $\mathcal{P}$, we have at most $2|V|$ iterations, and the algorithm ends. 
We note $\mathcal{P}^{(i)}, G_{0}^{(i)}=\left(V_{0}^{(i)}, E_{0}^{(i)}\right), c^{(i)}, g^{(i)}$ the values of $\mathcal{P}, G_{0}=\left(V_{0}, E_{0}\right), c, g$ (respectively) at the beginning of the $i$-th iteration. We also note $c^{(0)}$ the original bipartition (which is different from $c^{(1)}$ because of the switch of $c\left(x_{0}\right)$ ).

To prove the validity of the algorithm, we have to prove that:

- Error cannot be returned if $H$ is a Sterboul hypergraph.

- The output of the algorithm if no Error occurs is a bicoloration.

Before proving those points, we claim the following:

Claim 1 Suppose that $H$ is a Sterboul hypergraph. Consider the beginning of the $i$-th iteration. Let $\mathcal{P}^{(i)}=\left(x_{k} \ldots x_{0}\right)$, and $e_{j}=g^{(i)}\left(x_{j}\right)$ for $j=0, \ldots, k$. Then we have:

(a) For each $j=0, \ldots, k, x_{j}$ is the only vertex of its color in $e_{j}$.

(b) For each $j=0, \ldots, k-1$ we have $e_{j} \cap e_{j+1}=\left\{x_{j}\right\}$.

(c) Two non-consecutive edges are disjoint.

Proof: The proof works by induction on $i$.

For $i=1$ the claim clearly holds since $\mathcal{P}^{(1)}=\left(x_{0}\right)$.

We now consider $i \geq 1$ and we suppose the claim holds at iteration $i$. We are going to prove that it also holds at iteration $i+1$. Let $\mathcal{P}^{(i)}=\left(x_{k} \ldots x_{0}\right)$, and $e_{j}=g^{(i)}\left(x_{j}\right)$ for $j=0, \ldots, k$.

If during the $i$-th iteration the algorithm dropped $x_{k}$ out of $\mathcal{P}$ (that is $\mathcal{P}^{(i+1)}=\left(x_{k-1} \ldots x_{0}\right)$ ), the claim clearly holds at iteration $i+1$. Thus we assume that the algorithm found $e_{k+1} \in \mathcal{E}$ with $x_{k} \in e_{k+1}$ that is monochromatic for $c^{(i)}$, and $x_{k+1} \in e_{k+1} \backslash V_{0}^{(i)}$ (because we assume that there is a $(i+1)$-th iteration or else the claim is true) so that $\mathcal{P}^{(i+1)}=\left(x_{k+1} x_{k} \ldots x_{0}\right)$.

Since $(a)$ holds at iteration $i$, we know that if $w \in e_{k} \backslash x_{k}$ then $c^{(i)}(w) \neq c^{(i)}\left(x_{k}\right)$. As $x_{k} \in e_{k+1}$ and $e_{k+1}$ is monochromatic for $c^{(i)}$, then $e_{k} \cap e_{k+1}=\left\{x_{k}\right\}$ and $(b)$ holds at iteration $i+1$.

Suppose $j_{0}=\max \left\{0 \leq j \leq k-1 \mid e_{j} \cap e_{k+1} \neq \emptyset\right\}$ exists, and let $y \in e_{j_{0}} \cap e_{k+1}$. If $k-j_{0}$ is odd, then $\left(y, e_{j_{0}}, x_{j_{0}}, \ldots, e_{k+1}, y\right)$ is a Sterboul cycle (because $(c)$ holds at iteration $i$ and $(b)$ holds at iteration $i+1)$, so $k-j_{0}$ is even. But then since $(a)$ holds at iteration $i$, we have $c^{(i)}(y) \neq c^{(i)}\left(x_{j_{0}}\right)\left(y \neq x_{j_{0}}\right.$ by definition of $\left.j_{0}\right), c^{(i)}\left(x_{j_{0}}\right) \neq c^{(i)}\left(x_{j_{0}+1}\right), \ldots, c^{(i)}\left(x_{k-1}\right) \neq c^{(i)}\left(x_{k}\right)$ and then $c^{(i)}(y) \neq c^{(i)}\left(x_{k}\right)$, which is impossible because $e_{k+1}$ is monochromatic for $c^{(i)}$. Hence $j_{0}$ does not exist, and $(c)$ holds at iteration $i+1$.

Thus $x_{k+1} \notin e_{j}$ for all $j=0, \ldots, k$. Since the only color switch done during the $i$-th iteration concerns $x_{k+1}$, then $(a)$ holds at iteration $i+1$.

This achieves to prove the claim.

Now we are able to prove the validity of the algorithm.

- Suppose that $H$ is a Sterboul hypergraph. Consider an iteration $i$, and let $\mathcal{P}^{(i)}=\left(x_{k} \ldots x_{0}\right)$. If $k=1$ then from $(b)$ of the claim we have $x_{1} \notin e_{0}$. If $k \geq 2$ then from $(c)$ of the claim we also have $x_{k} \notin e_{0}$. This proves that we always have $e_{0} \cap V_{0}=\left\{x_{0}\right\}$.

If Error is returned, it means that at a given iteration $i_{0}$, the algorithm found an edge $e$ monochromatic for $c^{\left(i_{0}\right)}$ such that $e \backslash V_{0}^{\left(i_{0}\right)}=\emptyset$. Then $e$ was also monochromatic for $c^{(0)}$, but $e_{0}$ was the only such edge, 
so we have a contradiction because we have just seen that we must have $e_{0} \cap V_{0}^{\left(i_{0}\right)}=\left\{x_{0}\right\}$. Thus if $H$ is a Sterboul hypergraph, Error cannot be returned.

- Finally, if the bipartition obtained by the algorithm is not a bicoloration then we have some $e \in \mathcal{E}$ that is monochromatic. Consider $i_{0}$ the last iteration during which the algorithm dropped a vertex of $e$ out of $\mathcal{P}\left(i_{0}\right.$ exists or else $e$ was monochromatic with $c^{(0)}$, but $e_{0}$ was the only such edge and $e_{0}$ is not monochromatic at the end of the algorithm), and let $y_{0}$ be that vertex. Then $e$ was not monochromatic with $c^{\left(i_{0}\right)}$ or else the algorithm would have considered $e$ during the iteration $i_{0}$ instead of dropping $y_{0}$. But since no color switch concerning a vertex of $e$ occurs afterwards (by choice of $i_{0}$ ), we have a contradiction. Thus the final bipartition is a bicoloration.

So the algorithm is correct and the theorem is proved.

We can slightly modify the algorithm so that it gives a Sterboul cycle instead of just returning Error when the hypergraph is not Sterboul (in order to have a certificate that the hypergraph is not Sterboul).

To do so we just have to check at each iteration that the properties of Claim 1 still hold. If not it means that the monochromatic edge considered intersects the path induced by the stack, and a Sterboul cycle can be easily found.

Our algorithm finds a bicoloration for Sterboul hypergraphs in polynomial time. However it cannot be used to recognize bicolorable hypergraphs (since a Sterboul hypergraph may be bicolorable) and neither to recognize Sterboul hypergraphs (since it may happen that it gives a bicoloration for a hypergraph that is not Sterboul).

The problem of recognizing bicolorable hypergraphs is well-known to be NP-complete [4]. But we leave the following question open: what is the complexity of recognizing Sterboul hypergraphs?

\section{References}

[1] P. Duchet, Hypergraphs, in R. Graham, M. Grötschel, L. Lovász, editors, Handbook of Combinatorics (1995), chapter 7, 381-432.

[2] J.-C. Fournier, M. Las Vergnas, Une classe d'hypergraphes bichromatiques II, Discrete Mathematics 7 (1974) 99-106.

[3] J.-C. Fournier, M. Las Vergnas, A class of bichromatic hypergraphs, Annals of Discrete Mathematics 21 (1984) 21-27.

[4] L. Lovász, Coverings and colorings of hypergraphs, Proc. 4th Southeastern Conference on Combinatorics, Graph Theory, and Computing, Utilitas Mathematica Publishing, Winnipeg (1973) 3-12. 